\title{
APLICAÇÃO DA PROGRAMAÇÃO DINÂMICA NA SUBSTITUIÇÃO DE POVOAMENTOS FLORESTAIS ${ }^{1}$
}

\author{
Márcio Lopes da Silva², Rodrigo Firmino da Silva ${ }^{3}$ e Helio Garcia Leite ${ }^{2}$
}

\begin{abstract}
RESUMO - Objetivaram-se, neste estudo, desenvolver e aplicar dois modelos de programação dinâmica para decidir sobre a melhor opção de manejo de um povoamento florestal ao longo do horizonte de planejamento. Com os modelos, procurou-se maximizar os lucros através de uma relação de recorrência referente às receitas e aos custos ao longo dos anos a partir de um modelo tradicional de substituição de equipamentos. Os resultados de ambos os modelos indicaram, para a maioria das situações, como melhor opção não cortar povoamentos jovens, seguido de cortar e reformar ou cortar e conduzir a brotação para os povoamentos com idades mais avançadas, isso para todos os estágios ( $\operatorname{de} f 1 \mathrm{a} f 7$ ). A vantagem de se usar a PD, neste caso, é que esta ferramenta oferece ao planejador uma gama maior de alternativas na hora da tomada de decisão. Conclui-se que, quando uma empresa quer maximizar os lucros de um povoamento florestal, sem se preocupar com o horizonte de planejamento ou com a floresta regulada, deveria optar pela idade ótima de corte simples tradicionalmente conhecida como rotação econômica. Porém, se a empresa quer tomar decisões para um horizonte de planejamento definido e posteriormente deseja vender a terra e a floresta, as alternativas são muitas, pois a empresa pode optar por cortar agora ou postergar o corte, conduzir a brotação ou reformar. Nesse caso, o modelo de PD desenvolvido aqui pode apresentar tais alternativas e indicar a melhor.
\end{abstract}

Palavras-chave: Reflorestamento, otimização florestal e manejo florestal.

\section{APPLICATION OF DYNAMIC PROGRAMMING FOR FOREST STAND REPLACEMENT}

\begin{abstract}
This work aims to develop and apply two models of dynamic programming to decide the best option of management of a forest stand along the planning horizon. The models aimed to maximize profit through a relation of recurrence concerning revenues and costs along the years and it was constructed based on a traditional model of replacement equipment. The results of both the models indicate that the best option for most situations is not to cut the young stands, followed by cutting and remodeling or cutting and lead to shooting for the older stands for all the training periods ( $f$ 1through $f 7$ ). The advantage of using DP, in this in case, is that this tool offers the planner a wider gamma of decision-making alternatives. It was concluded that for a company to maximize forest stand profits, without being concerned about planning horizon or regulated forest, it would have to opt for the traditionally known economic rotation. However, if the company wants to make decisions for a definite horizon planning and later sell the land and the forest, the alternatives are many. Thus, the company can opt for cutting immediately or delaying the cut, perform the shootings or remodel. In this case, the DP model developed can present such alternatives and indicate the best options.
\end{abstract}

Keywords: Forest stand, forest optimization, forest and management.

\footnotetext{
${ }^{1}$ Recebido em 12.06.2006 e aceito para publicação em 15.05.2007.

${ }^{2}$ Departamento de Engenharia Florestal da Universidade Federal de Viçosa (UFV). E-mail: <marlosil @ufv.br>.

${ }^{3}$ Plantar S.A. Reflorestamentos, 30380-090 Belo Horizonte-MG. E-mail: <rodrigo_firmino@ @lantar.com.br>.
} 


\section{INTRODUÇÃO}

Dentre as atividades de manejo, que são previamente planejadas e orçadas dentro da empresas florestais, a reforma ou substituição dos povoamentos é uma das mais importantes, por proporcionar a continuidade da atividade florestal e requerer altos investimentos.

Para Souza et al. (2001) antes de se pensar na questão da reforma propriamente dita, o conhecimento da idade em que os povoamentos serão cortados é ponto de capital importância em qualquer trabalho envolvendo manejo florestal. Só há sentido econômico em determinar a idade ótima de substituição após a determinação da idade ótima de corte.

Rezende et al. (2001), relataram que a reforma pode ser implementada a qualquer tempo, porém, para se estabelecer uma regulação da floresta e manter um fluxo constante de produção de madeira, é necessário definir qual o intervalo entre os cortes de determinado talhão, ou seja, qual a idade ótima de se fazer o corte do alto fuste e das talhadias (brotações).

Segundo Silva (1990), reformar consiste na substituição total de povoamentos de baixo potencial produtivo, ou com produtividade abaixo da esperada, por um novo povoamento originado do plantio de mudas. Por sua vez, Rezende et al. (1987) definiram reforma e substituição para o caso de um povoamento florestal, sendo a substituição considerada quando o povoamento não produz de maneira satisfatória.

Outra situação é a condução da brotação de cepas, após o corte raso da floresta, que se aplica a espécies florestais que têm capacidade de regenerar vegetativamente. Esse sistema de condução de povoamentos florestais, denominado talhadia, é adequado à maioria das espécies do gênero Eucalyptus, que apresentam a capacidade de lançar brotos em razão das gemas adventícias presentes no tronco, os quais se desenvolvem e podem ser conduzidos para reconstituir o povoamento. Tal característica é desejável, pois a partir de um único plantio pode-se obter uma ou mais colheitas, com redução significativa de gastos com reforma. Entretanto, em muitas regiões brasileiras a produtividade de povoamentos de eucalipto em regime de talhadia tem sido inferior àquela observada no primeiro ciclo, o que tem levado muitas empresas florestais a optarem pela reforma ou substituição dos povoamentos (FARIA et al., 2002).
Após essas atividades, as empresas esperam que as receitas geradas ao final do projeto superem os custos e permitam novos investimentos. $\mathrm{O}$ aumento nas receitas de uma empresa se dá através do progresso tecnológico, que por sua vez é possível devido à introdução de clones melhorados e adaptados aos sítios, à utilização de novas técnicas de plantio e a tratos culturais, resultando no aumento da produtividade da floresta a cada ciclo (REZENDE et al., 2001).

Nesse contexto, pode-se perceber uma tendência entre as empresas florestais de se estruturarem operacional e administrativamente dentro da metodologia gerencial mais moderna, adotando novas tecnologias que permitam seu desenvolvimento e retornos financeiros melhorados (SOARES, 2002).

Visando à melhoria do planejamento florestal, a fim de auxiliar as tomadas de decisões nas atividades florestais, existem técnicas que visam a maximização do retorno financeiro. Entre essas técnicas está a pesquisa operacional, que é uma ciência aplicada voltada para a resolução ótima de problemas reais, desde os mais simples aos mais complexos (SOARES, 2002).

No setor florestal, os métodos de pesquisa operacional mais empregados são: programação linear, programação não-linear, programação dinâmica, programação por metas, simulação e redes de operação PERT-COM (LEITE, 1994). Mais especificamente, Silva (2000) relatou, que no planejamento florestal, as técnicas de programação linear, programação dinâmica e simulação têm sido empregadas com maior freqüência.

Dentre esses métodos, a programação dinâmica (PD) é útil em número substancial de aplicações no setor florestal. Trata-se de uma técnica de pesquisa operacional para otimização de funções lineares e nãolineares que se baseiam na decomposição do problema original em subproblemas ou estágios, podendo ser utilizada para resolver problemas que envolvem uma seqüência de intervenções ao longo do tempo, o que evidencia o potencial para aplicações florestais (DREYFUS e LAW, 1986).

A PD tem como principal vantagem, segundo Amidon e Akin (1968), a análise marginal tradicional para a solução de problemas de gestão em povoamentos eqüiâneos, os quais apresentam grande flexibilidade do modelo e alta eficiência da sensibilidade da solução ótima. 
Com relação ao uso da PD como ferramenta auxiliar para substituição de povoamentos florestais, são poucos os trabalhos e experiências. Mas podem-se citar, dentre outros, os trabalhos realizados por Ahrens (1992), que estudou, simultaneamente, rotação e desbastes ótimos; Leite (1994) desenvolveu um modelo de conversão de troncos em multiprodutos; Lima et al. (1997) desenvolveram um modelo de suporte à decisão sobre comércio e uso dos multiprodutos da madeira; Filgueiras (1997) trabalhou num modelo de substituição de equipamentos; Arce (1999) empregou ferramentas matemáticas de programação dinâmica na otimização do transporte de multiprodutos florestais; Chichorro (2000) utilizou um modelo de programação dinâmica e cadeia de Markov, para executar uma analise econômica de multiprodutos madeireiros de uma floresta natural; Soares (2002), utilizou um modelo matemático de programação dinâmica para otimização do uso da madeira em povoamentos de eucalipto; e Marques (2003) fez uso da programação dinâmica e de equações diferenciais para transformação e substituição de equipamentos.

A decisão de substituir ou não um povoamento nada mais é que a escolha de dois ou mais projetos a serem analisados do ponto de vista financeiro, considerando-se os custos e receitas futuras. A alternativa mais lucrativa por certo deve ser escolhida. A substituição de povoamentos florestais, seja tardia ou prematura, leva a empresa a incorrer em perdas financeiras. Ao decidir sobre a substituição do povoamento, pressupõese a definição e prévia análise do horizonte de planejamento, do futuro do investimento e dos custos operacionais. Dessa forma, a substituição de povoamentos florestais se mostra como uma atividade complexa, que resultará em importante tomada de decisão.

O objetivo geral deste estudo foi desenvolver e aplicar dois modelos de programação dinâmica para decidir sobre a melhor opção de manejo de um povoamento florestal ao longo do horizonte de planejamento.

\section{MATERIAL E MÉTODOS}

O modelo desenvolvido neste trabalho representa uma ferramenta para definir o momento ideal para a substituição de um único povoamento e não de uma floresta com talhões de diferentes idades. Esse modelo foi adaptado dos modelos existentes para substituição de equipamentos, como aqueles utilizados por Gupta e Cozzolino (1974), Filgueiras (1997) e Marques (2003). O objetivo dos dois primeiros autores era minimizar os custos operacionais, já o último apresentou um modelo de maximização de receitas e, portanto, mais próximo da situação de substituição de povoamentos florestais.

\subsection{Modelo de programação dinâmica para substituição de povoamentos}

O primeiro modelo apresentado aqui permite dicidir sobre não cortar a floresta (NC), mantendo o povoamento por mais um ano ou cortar e reformar (CR) a área, implantando-se um novo povoamento. A relação de recorrência estabelecida foi:

$f_{N}(n)=M A X$

$\left[R f_{n+1} *(1+i)^{-(n+1)}\right]-\left[C M_{n+1}\left(1+p_{n+1}\right) *(1+i)^{-(n+1)}\right]+$ $f_{N-1}(n+1)=$ não cortar

$\left[R f_{I} *(1+i)^{-1}\right]+\left[R n(1+i)^{-n}\right]-C R_{o}-\left[C M_{1}\left(1+p_{1}\right) *\right.$ $\left.(1+i)^{-1}\right]+f_{N-1}(1)=$ cortar e reformar

$\operatorname{Com} f_{o}(n)=R_{n} *(1+i)^{-n}$,

em que:

$\mathrm{f}_{\mathrm{N}}(\mathrm{n})=$ lucro (ou prejuízo) total atualizado dos $\mathrm{N}$ estágios futuros, tendo o povoamento em consideração $\mathrm{n}$ anos de idade no início do processo e sendo tomadas decisões ótimas em todos os futuros estágios (N);

$\boldsymbol{N}=$ número total de estágios ou tamanho do horizonte de planejamento;

$\boldsymbol{n}=$ idade do povoamento florestal com a qual o processo é iniciado, em anos, $\mathrm{n}=1,2, \ldots, \mathrm{N}$;

$\boldsymbol{C R}_{o}=$ custo de reforma ou implantação do novo povoamento florestal, em US\$/ha;

$\boldsymbol{C M _ { n }}=$ custo de manutenção anual do povoamento florestal no início do ano $\mathrm{n}, \mathrm{n}=1,2, \ldots, \mathrm{N}$, em US\$/ ha; sendo $\boldsymbol{C M}_{n}$ determinado pela soma dos custos anuais de combate à formiga, manutenção de estradas e aceiros e custo de oportunidade da terra;

$\boldsymbol{R} \boldsymbol{f}_{n}=$ receita do corte da floresta no ano $\mathrm{n}, \mathrm{n}=$ $1,2, \ldots \mathrm{N}$, em US\$/ha;

$\boldsymbol{R}_{n}=$ valor da floresta no final do ano $\mathrm{n}, \mathrm{n}=1$, $2, \ldots \mathrm{N}$, em US $\$ /$ ha;

$\boldsymbol{p}_{\boldsymbol{n}}=$ fator de custos anuais não-controláveis para determinado tipo de povoamento, expresso em termos porcentuais em relação a $\mathrm{CM}_{\mathrm{n}}$, que varia em função da idade do povoamento; e

$\boldsymbol{i}=$ taxa de desconto anual.

R. Árvore, Viçosa-MG, v.31, n.6, p.1063-1072, 2007 
Considerou-se que, no final do processo $\left(f_{o}(n)\right)$, ocorre a venda do povoamento estando ele no ponto de corte ou não, por isso $\mathrm{f}_{\mathrm{o}}(\mathrm{n})=\mathrm{R}_{\mathrm{n}} *(1+\mathrm{i})^{-\mathrm{n}}$.

A decisão econômica ótima é tomada comparandose os valores da relação de recorrência referentes a manter o povoamento por mais um ano ou substituir, isto é, o povoamento é mantido quando: o valor do povoamento no ano seguinte menos o custo de manutenção do próximo ano for maior que a venda da floresta mais o valor da nova floresta implantada menos o custo de manutenção dessa nova floresta; ou, ainda, a decisão de se postergar o corte por mais um período for mais lucrativa que se cortar a floresta no ano n e se implantar uma nova floresta. Caso contrário, o povoamento deve ser substituído.

\subsection{Modelo com programação dinâmica incluindo a opção de condução da brotação}

Neste modelo, tem-se a opção de manter a floresta por mais um período (NC), cortar e reformar (CR) ou cortar e conduzir a brotação (CCB). Pode-se, então, escrever a seguinte relação de recorrência:

$f_{N}(n)=M A X$

$\left[R f_{n+1} *(1+i)^{-(n+1)}\right]-\left[C M_{n+1}\left(1+p_{n+1}\right) *(1+i)^{-(n+1)}\right]+$ $f_{N-1}(n+1)=(N C)$

$\left[R f_{1} *(1+i)^{-1}\right]+\left[R n(1+i)^{-n}\right]-C R_{o}-\left[C M_{1}\left(1+p_{1}\right) *(1+i)^{-}\right.$

$\left.{ }^{1}\right]+f_{N-1}(1)=(C R)$

$\left[R f b_{1} *(1+i)^{-1}\right]+\left[R n(1+i)^{-n}\right]-\left[C B_{1}\left(1+p_{1}\right) *(1+i)^{-}\right.$

$\left.{ }^{1}\right]+f_{N-1}(1)=(C C B)$

$\operatorname{Com} f_{o}(n)=R_{n} *(1+i)^{-n}$,

em que:

$\boldsymbol{C B}_{o}=$ custo de condução de brotação da floresta que foi cortada, em US\$/ha;

$\boldsymbol{R} \boldsymbol{f} \boldsymbol{b}_{\boldsymbol{n}}=$ valor da floresta brotada no ano $\mathrm{n}, \mathrm{n}=$ $1,2, \ldots \mathrm{N}$, em US $\$ / \mathrm{ha}$; as demais variáveis foram definidas conforme anteriormente.

\subsection{Estudo de caso}

\section{Produção florestal}

Para aplicar o modelo de PD foi necessário obter a produção florestal. Para tanto, utilizou-se um modelo de crescimento e produção (modelo de Clutter), ajustado para uma situação real por Soares (1999), com o híbrido Eucalyptus grandis x Eucalyptus urophylla, produzido a partir de estacas, considerando a idade-índice de 60 meses. Esse modelo é apresentado no Quadro 1. Utilizou-se, neste estudo, um índice de local de $24 \mathrm{e}$ área basal inicial de $13 \mathrm{~m}^{2} / \mathrm{ha}$.

Para obter a produção da segunda talhadia, considerou-se um decréscimo de $10 \%$ em relação ao plantio original.

\section{Custos e receitas}

Os custos e preços utilizados nesta análise são apresentados no Quadro 2, cujoss valores correspondem a dados médios praticados pelas empresas florestais. Para a análise ficar mais próxima da realidade, considerouse que o preço da madeira varia em função da idade da floresta. Todo o modelo foi desenvolvido em planilha eletrônica. Os valores foram obtidos em fevereiro de 2006, quando US\$1,00 correspondia a $\mathrm{R} \$ 2,15$.

Quadro 1 - Modelos de crescimento e produção (Modelo de Clutter) genérico e ajustado por Soares (1999), para uma situação real

Table 1 - Growth and production model (Clutter's Model) adjusted by Soares (1999), for a real situation

\begin{tabular}{|c|}
\hline Modelo genérico \\
\hline $\begin{array}{c}\ln \mathrm{V}_{2}=\mathrm{b}_{0}+\mathrm{b}_{1 *}\left(1 / \mathrm{I}_{2}\right)+\mathrm{b} 2 * \mathrm{~S}+\mathrm{b}_{3 *} \ln \left(\mathrm{B}_{2}\right) \\
\ln \mathrm{B}_{2}=\ln \mathrm{B}_{1 *}\left(\mathrm{I}_{1} / \mathrm{I}_{2}\right)+\mathrm{a}_{0 *}\left(1-\mathrm{I}_{1} / \mathrm{I}_{2}\right)+\mathrm{a}_{1 *}(\mathrm{~S})_{*}\left(1-\mathrm{I}_{1} / \mathrm{I}_{2}\right)\end{array}$ \\
\hline Modelo ajustado \\
\hline $\begin{array}{l}\mathrm{LnV}_{2}=1,81969-25,712511 / \mathrm{I}_{2}+0,02073_{*} \mathrm{~S}+1,13070_{*} \ln \mathrm{B} \\
\mathrm{nB}_{2}=\ln \mathrm{B}_{1 *}\left(\mathrm{I}_{1} / \mathrm{I}_{2}\right)+2,96228_{*}\left(1-\mathrm{I}_{1} / \mathrm{I}_{2}\right)+0,01533_{*} \mathrm{~S}_{*}\left(1-\mathrm{I}_{1} / \mathrm{I}\right.\end{array}$ \\
\hline
\end{tabular}

em que: $\ln =\log$ aritmo neperiano; $\mathrm{S}=$ índice de local, em $\mathrm{m} ; \mathrm{B}$

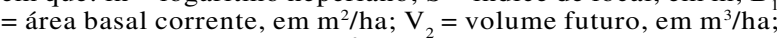
$\mathrm{B}_{2}=$ área basal futura, em ${ }^{2} / \mathrm{ha} ; \mathrm{I}_{1}^{2}=$ idade atual, em meses; $\mathrm{I}_{2}$ $=$ idade futura, em meses; $\mathrm{e}_{0} \mathrm{a} \mathrm{b}_{3} ; \mathrm{a}_{0}$ e $\mathrm{a}_{1}=$ parâmetros do modelo na forma estrutural.

Quadro 2 - Itens de custo e receita das atividades florestais Table 2 - Cost and revenue items for forest activities

\begin{tabular}{|c|c|}
\hline Variável & Valor \\
\hline Preço da madeira & US\$ $18,00 / \mathrm{m}^{3}$ \\
\hline Taxa de crescimento do preço & $5 \%$ a. a. \\
\hline Custo de implantação & US\$ $1.00,00 / \mathrm{ha}$ \\
\hline Valor da terra & US\$ 400,00/ha \\
\hline Custo anual da terra & US\$ $\$ 40,00 /$ ha \\
\hline Custo anual & US\$ $37,00 /$ ha \\
\hline Custo de $1^{a}$ manutenção & US $\$ 300,00 / \mathrm{ha}$ \\
\hline Custo de $2^{\mathrm{a}}$ manutenção & US\$200,00/ha \\
\hline Custo de $1^{\text {a }}$ condução da brotação & US\$300,00/ha \\
\hline Custo de $2^{\mathrm{a}}$ condução da brotação & US\$300,00/ha \\
\hline Taxa de juros & $10 \%$ a. a. \\
\hline
\end{tabular}




\section{Custos e receitas atuais}

Como os custos e receitas da floresta ocorrem em diferentes anos, torna-se necessário corrigir seus valores para uma mesma base temporal, pela taxa de juros. Esses valores são os custos e receitas atuais.

\section{Valor Presente Líquido (VPL)}

O VPL é a diferença entre o valor atual da receita menos o valor atual do custo do projeto. O VPL marginal é o acréscimo (ou variação) do VPL de um ano pra outro. O VPL marginal igual a zero indica o ponto ótimo ou a idade ótima econômica de corte.

\section{RESULTADOS E DISCUSSÃO}

\subsection{Produção e preço da madeira}

A Figura 1 ilustra a produção de um povoamento florestal do plantio original (alto fuste) e da brotação (segunda rotação). Nota-se que o povoamento em $1^{a}$ rotação apresentou maior produção, em comparação com um povoamento de $2^{\text {a }}$ rotação, que passou pela condução de brotação, podendo, assim, ter ocorrido perdas por mortalidade, competição ou desbrota.

Na Figura 2 éilustrada a tendência do preço da madeira em função da idade do povoamento. $O$ fato de considerar o preço variável em função da idade do povoamento torna o estudo mais interessante e mais próximo da realidade do mercado de madeira atual. Uma vez que um povoamento com mais idade tende a agregar mais valor comercial, ou seja, as árvores possuem diâmetro e altura maiores e, conseqüentemente, maior volume comercial, além de poderem apresentar características tecnológicas desejáveis, dependendo da finalidade da floresta.

\subsection{Custos, receitas e Valor Presente Líquido (VPL)}

A partir da função de produção florestal e dos itens custo das atividades florestais (Quadros 1 e 2), foram elaborados os Quadros 3 e 4, em que são apresentados os valores de custo, receita e Valor Presente Líquido (VPL) para a primeira e segunda rotações, respectivamente, em função da idade do povoamento.

Nos Quadros 3 e 4, pode-se observar que a idade ótima econômica de corte da primeira e da segunda rotação foi de nove anos, pois ela ocorre onde o VLP é máximo e o VPL marginal é zero. Essa rotação está além daquelas comumente encontradas de sete anos, porque na análise deste estudo considerou-se que o preço varia em função da idade do povoamento (Figura 2), postergando um pouco o corte. Com esses mesmos dados e levando em conta o preço constante, a rotação econômica diminui para os sete anos, o que indica que os dados estão coerentes com a maioria das empresas florestais.

Quando uma empresa quer maximizar os lucros de um povoamento florestal, sem se preocupar com o horizonte de planejamento ou com a floresta regulada, ela deve optar pela idade ótima de corte tradicional, referida anteriormente. Porém, se a empresa quer tomar decisões para um horizonte de planejamento menor e posteriormente vai vender a terra e a floresta, as alternativas são muitas, pois a empresa pode optar por cortar agora ou postergar o corte, conduzir a brotação ou reformar. Nesse caso, o modelo de PD desenvolvido aqui pode apresentar tais alternativas e indicar a melhor delas, conforme tratado a seguir.

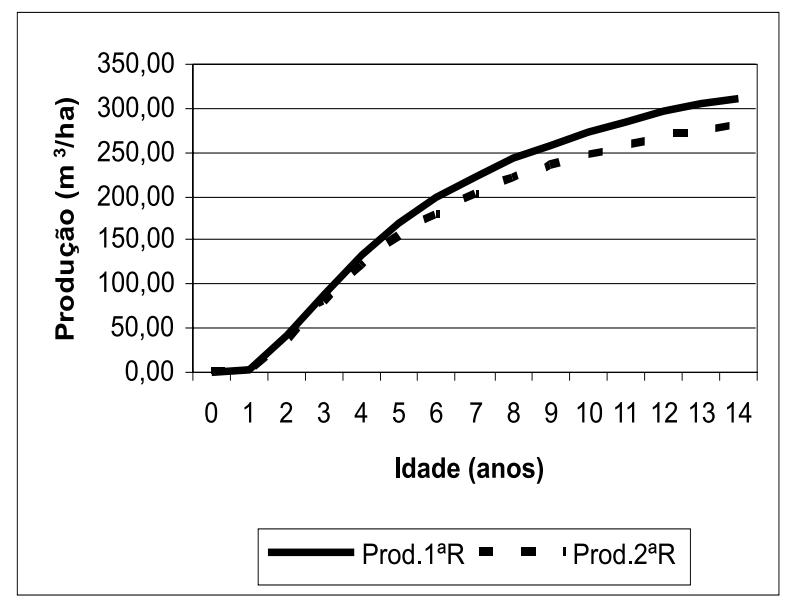

Figura 1 - Produção para a $1^{\mathrm{a}} \mathrm{e} 2^{\mathrm{a}}$ rotações em um povoamento florestal.

Figure 1 - Production for the first and second rotations in a forest stand.

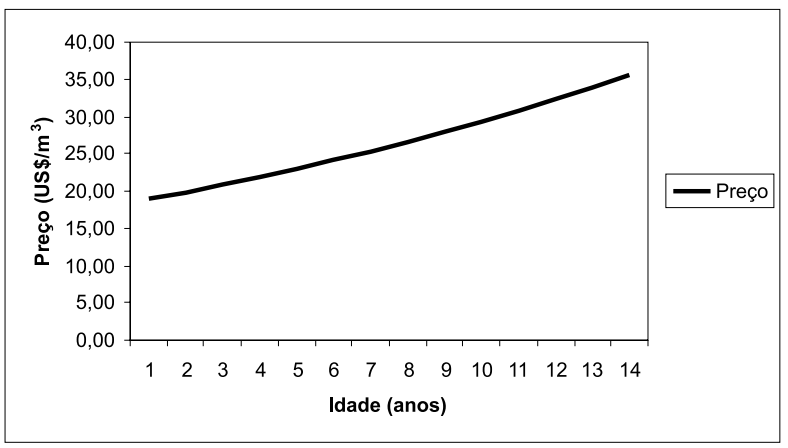

Figura 2 - Preço da madeira em função da idade do povoamento florestal.

Figure 2 - Wood price in function of forest stand age. 
Quadro 3 - Operações, custos, receitas e VPL (US\$/ha) para a $1^{\text {a }}$ rotação de um povoamento florestal Table 3 -Operations, costs, revenues, and NPV (US\$/ha) for the first rotation of a forest stand

\begin{tabular}{|c|c|c|c|c|c|c|c|c|c|c|}
\hline \multirow[t]{2}{*}{$\overline{\text { Ano }}$} & \multirow[t]{2}{*}{ Operação } & \multirow[t]{2}{*}{ Custo $^{1}$} & \multicolumn{2}{|c|}{$1^{\mathrm{a}}$ rotação } & \multirow{2}{*}{$\begin{array}{c}\text { Receita } \\
\text { atual }\end{array}$} & \multirow{2}{*}{$\begin{array}{c}\text { Receita } \\
\text { anual atual }\end{array}$} & \multirow{2}{*}{$\begin{array}{c}\text { Custo } \\
\text { anual atual } \\
\end{array}$} & \multirow{2}{*}{$\begin{array}{c}\text { Custo } \\
\text { acumulado } \\
\end{array}$} & \multirow{2}{*}{$\begin{array}{c}\mathrm{VPL} \\
\text { (Receita-Custo) }\end{array}$} & \multirow{2}{*}{$\begin{array}{c}\mathrm{VPL} \\
\text { marginal }\end{array}$} \\
\hline & & & Produção & Receita & & & & & & \\
\hline 0 & Plantio & 1000,00 & 0,00 & 0,00 & 0,00 & 0,00 & 1000,00 & 1000,00 & $-1000,00$ & $-1000,00$ \\
\hline 1 & 1a. Manut. & 377,00 & 3,83 & 72,48 & 65,89 & 65,89 & 342,73 & 1342,73 & $-1276,84$ & $-276,84$ \\
\hline 2 & 2a. Manut. & 277,00 & 40,99 & 813,51 & 672,32 & 606,43 & 228,93 & 1571,65 & $-899,33$ & 377,51 \\
\hline 3 & 3a. Manut. & 77,00 & 90,30 & 1881,65 & 1413,71 & 741,39 & 57,85 & 1629,50 & $-215,79$ & 683,54 \\
\hline 4 & 4a. Manut. & 77,00 & 134,03 & 2932,38 & 2002,86 & 589,15 & 52,59 & 1682,10 & 320,76 & 536,55 \\
\hline 5 & 5a. Manut. & 77,00 & 169,86 & 3902,16 & 2422,94 & 420,08 & 47,81 & 1729,91 & 693,03 & 372,27 \\
\hline 6 & 6a. Manut. & 77,00 & 198,92 & 4798,35 & 2708,54 & 285,61 & 43,46 & 1773,37 & 935,17 & 242,14 \\
\hline 7 & 7a. Manut. & 77,00 & 222,68 & 5640,00 & 2894,21 & 185,67 & 39,51 & 1812,88 & 1081,33 & 146,16 \\
\hline 8 & 8a. Manut. & 77,00 & 242,34 & 6444,91 & 3006,60 & 112,39 & 35,92 & 1848,81 & 1157,79 & 76,46 \\
\hline 9 & 9a. Manut. & 77,00 & 258,83 & 7227,50 & 3065,17 & 58,57 & 32,66 & 1881,46 & 1183,70 & 25,91 \\
\hline 10 & 10a. Manut. & 77,00 & 272,82 & 7999,14 & 3084,01 & 18,85 & 29,69 & 1911,15 & 1172,87 & $-10,84$ \\
\hline 11 & 11a. Manut. & 77,00 & 284,83 & 8768,81 & 3073,41 & $-10,60$ & 26,99 & 1938,14 & 1135,28 & $-37,59$ \\
\hline 12 & 12a. Manut. & 77,00 & 295,24 & 9543,78 & 3040,94 & $-32,47$ & 24,53 & 1962,67 & 1078,27 & $-57,01$ \\
\hline 13 & 13a. Manut. & 77,00 & 304,35 & 10330,02 & 2992,24 & $-48,70$ & 22,30 & 1984,97 & 1007,26 & $-71,01$ \\
\hline 14 & 14a. Manut. & 77,00 & 312,37 & 11132,63 & 2931,57 & $-60,67$ & 20,28 & 2005,25 & 926,32 & $-80,95$ \\
\hline
\end{tabular}

${ }^{1}$ Custos, receitas e VPL em US $\$ /$ ha; e ${ }^{2}$ Produção em $\mathrm{m}^{3} / \mathrm{ha}$.

Quadro 4 - Operações, custos, receitas e VPL para a $2^{\text {a }}$ rotação de um povoamento florestal Table 4-Operations, costs, revenues, and NPV (US\$/ha) for the second rotation of a forest stand

\begin{tabular}{|c|c|c|c|c|c|c|c|c|c|c|}
\hline \multirow[t]{2}{*}{ Ano } & \multirow[t]{2}{*}{ Operação } & \multirow{2}{*}{$\begin{array}{l}\text { Custo }^{1} \\
\text { US\$/ha }\end{array}$} & \multicolumn{2}{|c|}{$2^{\mathrm{a}}$ rotação } & \multirow{2}{*}{$\begin{array}{c}\text { Receita } \\
\text { atual }\end{array}$} & \multirow{2}{*}{$\begin{array}{c}\text { Receita } \\
\text { anual atual }\end{array}$} & \multirow{2}{*}{$\begin{array}{c}\text { Custo } \\
\text { anual atual }\end{array}$} & \multirow{2}{*}{$\begin{array}{c}\text { Custo } \\
\text { acumulado }\end{array}$} & \multirow{2}{*}{$\begin{array}{c}\text { VPL } \\
\text { (Receita-Custo) }\end{array}$} & \multirow{2}{*}{$\begin{array}{c}\text { VPL } \\
\text { marginal }\end{array}$} \\
\hline & & & Produção ${ }^{2}$ & Receita & & & & & & \\
\hline 0 & & 0,00 & 0,00 & 0,00 & 0,00 & 0,00 & 0,00 & 0,00 & 0,00 & 0,00 \\
\hline 1 & 1Cond.Br. & 377,00 & 3,45 & 65,23 & 59,30 & 59,30 & 342,73 & 342,73 & $-283,43$ & $-283,43$ \\
\hline 2 & 2 Cond.Br. & 377,00 & 36,89 & 732,16 & 605,09 & 545,79 & 311,57 & 654,30 & $-49,21$ & 234,22 \\
\hline 3 & 3a. Manut. & 77,00 & 81,27 & 1693,48 & 1272,34 & 667,25 & 57,85 & 712,15 & 560,19 & 609,40 \\
\hline 4 & 4a. Manut. & 77,00 & 120,62 & 2639,14 & 1802,57 & 530,23 & 52,59 & 764,74 & 1037,83 & 477,64 \\
\hline 5 & 5a. Manut. & 77,00 & 152,87 & 3511,95 & 2180,64 & 378,07 & 47,81 & 812,55 & 1368,09 & 330,26 \\
\hline 6 & 6a. Manut. & 77,00 & 179,03 & 4318,51 & 2437,69 & 257,05 & 43,46 & 856,02 & 1581,67 & 213,58 \\
\hline 7 & 7a. Manut. & 77,00 & 200,41 & 5076,00 & 2604,79 & 167,10 & 39,51 & 895,53 & 1709,26 & 127,59 \\
\hline 8 & 8a. Manut. & 77,00 & 218,11 & 5800,42 & 2705,94 & 101,15 & 35,92 & 931,45 & 1774,49 & 65,23 \\
\hline 9 & 9a. Manut. & 77,00 & 232,95 & 6504,75 & 2758,65 & 52,71 & 32,66 & 964,11 & 1794,54 & 20,06 \\
\hline 10 & 10a. Manut. & 77,00 & 245,54 & 7199,22 & 2775,61 & 16,96 & 29,69 & 993,79 & 1781,82 & $-12,72$ \\
\hline 11 & 11a. Manut. & 77,00 & 256,35 & 7891,93 & 2766,07 & $-9,54$ & 26,99 & 1020,78 & 1745,29 & $-36,53$ \\
\hline 12 & 12a. Manut. & 77,00 & 265,72 & 8589,40 & 2736,85 & $-29,23$ & 24,53 & 1045,32 & 1691,53 & $-53,76$ \\
\hline 13 & 13a. Manut. & 77,00 & 273,91 & 9297,02 & 2693,02 & $-43,83$ & 22,30 & 1067,62 & 1625,40 & $-66,14$ \\
\hline 14 & 14a. Manut. & 77,00 & 281,14 & 10019,37 & 2638,41 & $-54,60$ & 20,28 & 1087,90 & 1550,52 & $-74,88$ \\
\hline
\end{tabular}

${ }^{1}$ Custos, receitas e VPL em US\$/ha; e ${ }^{2}$ Produção em m $3 /$ ha.

A partir dos dados dos Quadros 3 e 4 e da taxa de juros de $10 \%$ a.a., obtiveram-se os resultados para os modelos de PD apresentados nos Quadros 5 e 6. No Quadro 5 há referência ao modelo com duas alternativas: manter a floresta crescendo por mais um ano (NC) ou cortar e reformar a floresta (CR) para os diferentes horizontes de planejamento. Por exemplo, considerando que a empresa vai utilizar a terra por mais três anos (f3) e a floresta está com quatro anos de idade, a melhor opção seria (NC), ou seja, deixála crescendo por mais um período. Caso a floresta esteja com cinco anos de idade, a melhor opção seria (CR).

R. Árvore, Viçosa-MG, v.31, n.6, p.1063-1072, 2007 
Nesse caso, para a maioria das situações a melhor opção foi (NC) para povoamentos mais jovens, seguida de CR para povoamentos com idades mais avançadas, isso para todos os estágios (de $f 1$ a $f 7$ ).

No Quadro 6 há referência ao modelo com três alternativas: manter a floresta crescendo por mais um ano (NC), cortar e reformar a floresta (CR) e, ainda, cortar e conduzir a brotação (CCB). Os resultados indicam como as melhores opções, para cada horizonte (de $f$ 1 a $f 7),(\mathrm{NC})$ e (CCB), mas não aparece a opção (CR), pois o custo para conduzir a brotação, considerado no estudo, foi bem inferior ao de reforma ou de se implantar a floresta novamente. Quando a diferença entre o custo de condução da brotação e o custo de reforma é pequena, o modelo indica, em alguns casos, a opção (CR) como a melhor.

Comparando os dois modelos, observou-se que foram apresentados resultados semelhantes, prevalecendo as opções de não cortar o povoamento ainda jovem, cortar e reformar ou cortar e conduzir a brotação do povoamento mais velho.

A partir do Quadro 5, confeccionou-se um diagrama ou "árvore de decisão" (Figura 3), a fim de ilustrar a melhor decisão para um povoamento com sete anos de idade e horizonte de planejamento (estágio) de três anos, ou seja, o tempo em que a empresa pretende ter a posse da terra ou da floresta é de três anos. Portanto, a melhor política seria cortar e reformar (CR) esse povoamento com sete anos, e nos anos seguintes a floresta não seria cortada (NC) sendo vendidas a terra e a floresta em pé com três anos de idade.

A Figura 4 foi gerada a partir do Quadro 6 e ilustra a melhor alternativa, considerando-se além das opções (NC) e (CR), a opção (CCB). Nesse caso, uma empresa que teria um povoamento com sete anos e pretende otimizar o uso da terra por mais três anos teria como melhor política: cortar e conduzir a brotação (CCB) e não cortar (NC) a floresta nos dois últimos anos, ou seja, venderia a terra e a floresta com três anos. Na Figura 4 é possível visualizar também uma série de opções possíveis. Observa-se que, após cortar e conduzir a brotação, passa-se a ter somente as opções (NC) e (CR), pois foram consideradas apenas a primeira e a segunda rotação. A opção de (CCB) por duas vezes consecutivas, apesar de ser possível no modelo, foi descartada, pois, atualmente, as empresas têm utilizado no máximo dois cortes.

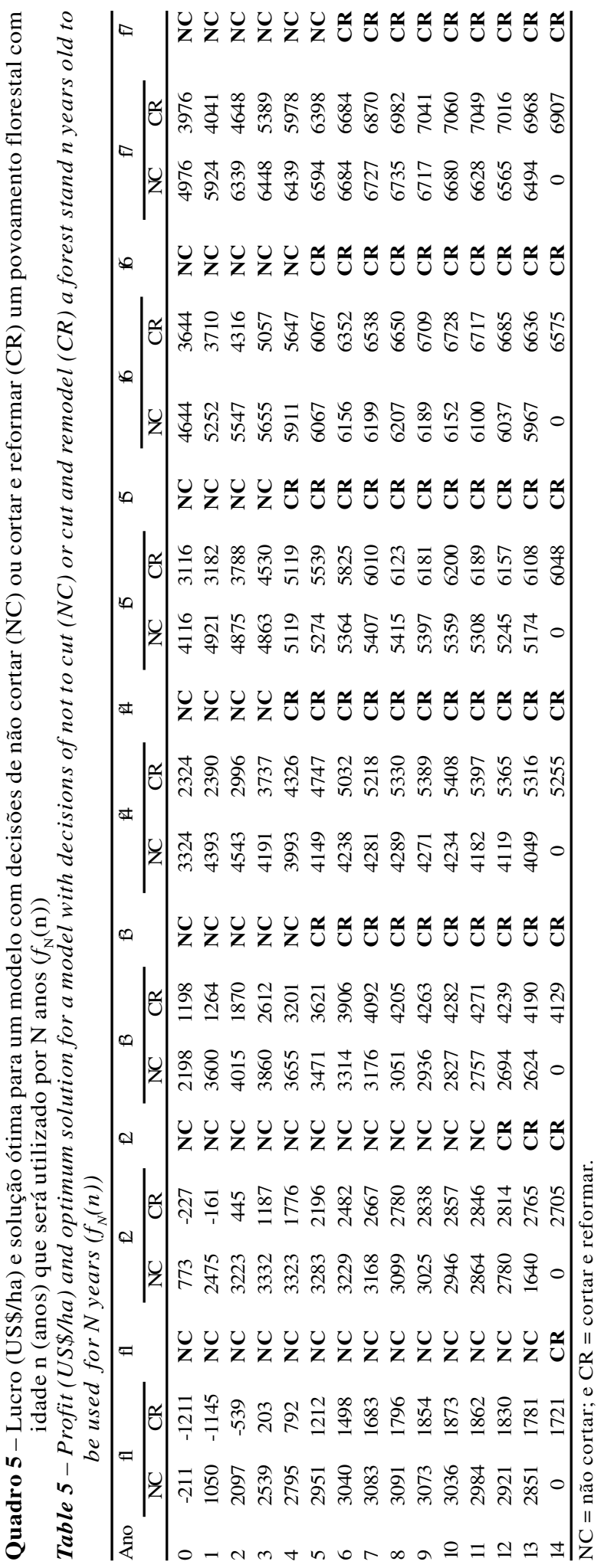

R. Árvore, Viçosa-MG, v.31, n.6, p.1063-1072, 2007 


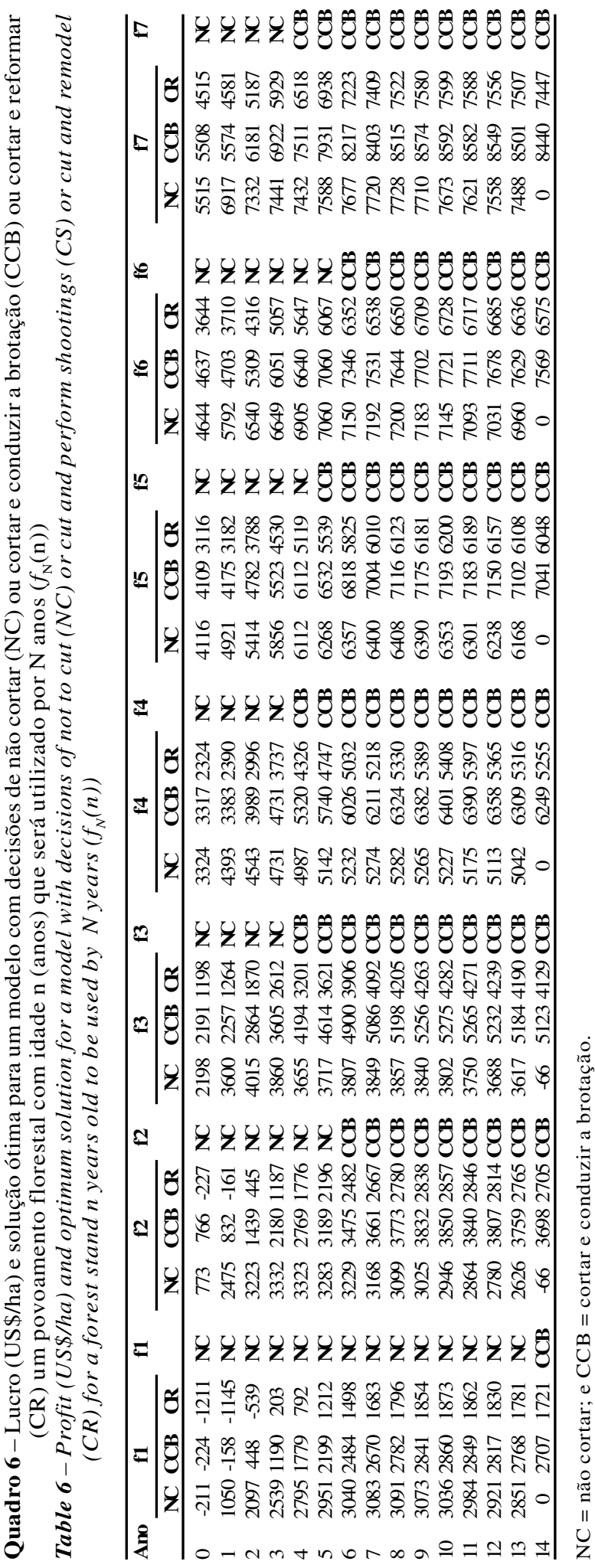

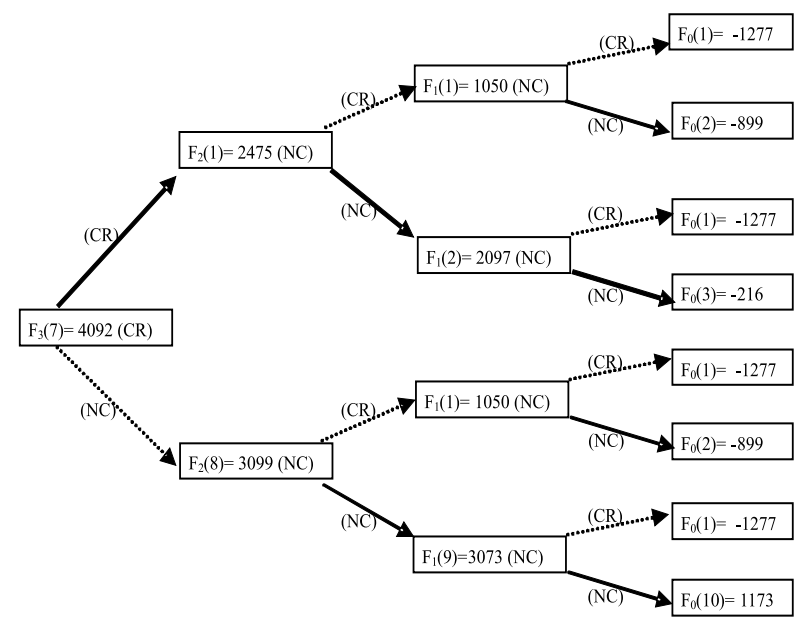

Figura 3 - Árvore de decisão e caminho ótimo do modelo de programação dinâmica com opções de não cortar (NC) ou cortar e reformar (CR), a partir de um povoamento com 7 anos idade, em que a terra seria utilizada por mais três anos.

Figure 3 -Decision tree and optimum path of the DP model with options of not to cut (NC) or to cut and remodel $(C R)$, from a seven year old stand whose land would be used for more three years.

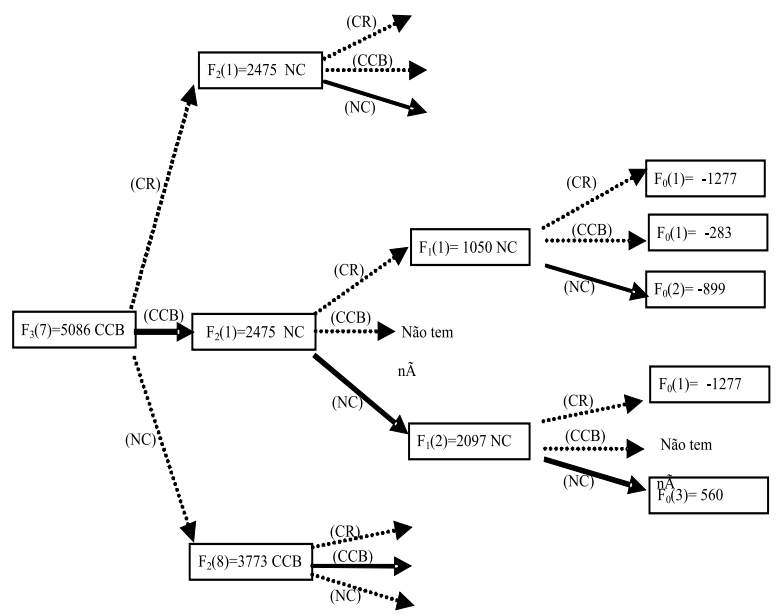

Figura 4 - Árvore de decisão e caminho ótimo do modelo de programação dinâmica, com opções de não cortar (NC), cortar e reformar (CR) e cortar e conduzir brotação (CCB), a partir de um povoamento com 7 anos idade, em que a terra seria utilizada por mais três anos.

Figure 4-Decision tree and optimum path of the dynamic programming model with options of not to cut (NC) or to cut and remodel (CR) and cut and conduct shootings (CS), from a seven- year-old stand whose land would be used for more three years. 


\section{CONCLUSÕES}

- Ambos os modelos geraram resultados semelhantes, ou seja, de não cortar o povoamento em idades jovens e cortar e reformar ou cortar e conduzir a brotação nas idades avançadas.

- Com o desenvolvimento dos modelos foi possível oferecer decisões ótimas de não cortar, reformar ou conduzir a brotação considerando um único povoamento florestal para um horizonte de planejamento pré-fixado.

- O modelo não otimiza a melhor opção para uma floresta com vários talhões com diferentes idades. Para essa última situação, recomenda-se utilizar outros modelos de planejamento florestal, como os Modelos I e II de programação linear.

- Há um enorme potencial para aplicação da PD no manejo de povoamentos florestais, ressaltandose que os modelos podem ser implementados em planilhas eletrônicas sem maiores problemas.

\section{REFERÊNCIAS}

AHRENS, S. A. Seleção simultânea do ótimo regime de desbastes e da idade de rotação, para povoamentos de Pinus taeda L., através de um modelo de programação dinâmica. 1992. 189f. Tese (Doutorado em Ciência Florestal) - Universidade Federal do Paraná, Curitiba, 1992.

AMIDON, E. L.; AKIN, G. S. Dynamic programming to determine optimum levels of growing stock. Forest Science, v.14, n.3, p.278-291, 1968.

ARCE, J. E. Um sistema de programação de transporte de multiprodutos florestais visando a minimização do custo da matéria-prima. 1999. 98f. Dissertação (Mestrado em Engenharia Florestal) Universidade Federal do Paraná, Curitiba, 1999.

CHICHORRO, J. F. Análise estrutural e econômica de multiprodutos da madeira. 2000. 241f. Tese (Doutorado em Ciência Florestal) - Universidade Federal de Viçosa, Viçosa, MG, 2000.

DREYFUS, S. E.; LAW, A. A. The art and theory of dynamic programming. Orlando: Academic Press, 1986. 318p.
FARIA, G. E. et al. Produção e estado nutricional de povoamento de Eucalyptus grandis, em segunda rotação, em resposta à adubação potássica. Revista Árvore, v.26, n.5, p.577-584, 2002.

FILGUEIRAS, J. F. Um modelo de substituição de equipamentos para minimizar custos operacionais em uma empresa florestal. 1997. 54f. Dissertação (Mestrado em Ciência) - Universidade Federal de Viçosa, Viçosa, MG, 1997.

GUPTA, S. K.; COZZOLINO, J. M.

Fundamentals of operations research for management: An introduction to quantitative methods. San Francisco: Holden Day, 1974. 405p.

LEITE, H. G. Conversão de troncos em multiprodutos da madeira, utilizandose programação dinâmica. 1994. 230f. Tese (Doutorado em Ciência Florestal) - Universidade Federal de Viçosa, Viçosa, MG, 1994.

LIMA, D. G. et al. Um modelo de suporte à decisão sobre multiprodutos de povoamentos florestais. Revista Árvore, v.21, n.1, p.35-48, 1997.

\section{MARQUES, G. M. Transformação e} substituição de equipamentos utilizando equações diferenciais e programação dinâmica. 2003. $48 \mathrm{f}$. Dissertação (Mestrado em Ciência Florestal)Universidade Federal de Viçosa, Viçosa, MG, 2003.

REZENDE, J. L. P.; SOUZA, A. N.; OLIVEIRA, A. D. Estudo do momento ótimo de substituição de povoamentos de Eucalyptus spp: o caso do aumento das receitas. Scientia Forestalis, n.59, p. 27-39, 2001.

REZENDE, J. L. P.; PAULA JUNIOR, G. G., RIBEIRO, G. A. Técnicas de análises econômicas usadas na tomada de decisão referentes à reforma de eucaliptais. In: SEMINÁRIO SOBRE ASPECTOS TÉCNICOS E ECONÔMICOS DA REFORMADE EUCALIPTAIS, 1987, Belo Horizonte. Anais... Belo Horizonte: Universidade Federal de Viçosa/Sociedade de Investigação Florestal, 1987. 82p.

R. Árvore, Viçosa-MG, v.31, n.6, p.1063-1072, 2007 
SILVA, A. A. L. Análise econômica da substituição de povoamentos de Eucalyptus spp. 1990. 109f. Dissertação (Mestrado em Ciência Florestal) - Universidade Federal de Viçosa, Viçosa, MG, 1990.

SILVA, G. F. Problemas no uso de programação matemática e simulação em regulação florestal. 2001. 89f. Tese (Doutorado em Ciência Florestal) - Universidade Federal de Viçosa, Viçosa, MG, 2001.
SOARES, T. S. Otimização do uso da madeira em povoamentos de eucalipto. 2002. 49f. Dissertação (Mestrado em Ciência Florestal) - Universidade Federal de Viçosa, Viçosa, MG, 2002.

SOUZA, A. N.; REZENDE, J. L. P.; OLIVEIRA, A. D. Momento ótimo de substituição de povoamentos de Eucalyptus spp - o caso da tecnologia constante. Revista Cerne, v.7, n.2, p.93-103, 2001. 